\title{
Percolation-Based Risk Index for Pathogen Invasion: Application to Soilborne Disease in Propagation Systems
}

\author{
S. Poggi, F. M. Neri, V. Deytieux, A. Bates, W. Otten, C. A. Gilligan, and D. J. Bailey
}

First and seventh authors: INRA, UMR1349 IGEPP, F-35653 Le Rheu, France; second, third, fourth, sixth, and seventh authors: Department of Plant Sciences, University of Cambridge, Downing Street, Cambridge, CB2 3EA, UK; third author: INRA, UE115 Domaine Expérimental d'Epoisses, F-21110 Bretenière, France; and fifth author: University of Abertay, SIMBIOS, Dundee, DD1 1HG, UK. Accepted for publication 17 April 2013.

\begin{abstract}
Poggi, S., Neri, F. M., Deytieux, V., Bates, A., Otten, W., Gilligan, C. A., and Bailey, D. J. 2013. Percolation-based risk index for pathogen invasion: Application to soilborne disease in propagation systems. Phytopathology 103:1012-1019.

Propagation systems for seedling growth play a major role in agriculture, and in notable cases (such as organic systems), are under constant threat from soil and seedborne fungal plant pathogens such as Rhizoctonia solani or Pythium spp. Yet, to date little is known that links the risk of disease invasion to the host density, which is an agronomic characteristic that can be readily controlled. We introduce here, for the first time in an agronomic system, a percolation framework to analyze the link. We set up an experiment to study the spread of the ubiquitous fungus $R$. solani
\end{abstract}

ABSTRACT

Field vegetables represent one of the largest crop production sectors of the horticultural industry, covering 245,000 ha with an approximate value of $€ 3.1$ billion in France in 2008 (15). The corresponding values for the United Kingdom in 2009 were 125,600 ha and $£ 0.80$ billion (10). For many horticultural crops the production of plants involves the growth, or propagation, of seedlings on a lattice in commercial propagation trays. Cell or plug propagation trays are often used for early crops that are subsequently transplanted into the field. These trays consist of individual cells or plugs, separated from each other by a thin wall. Sowing density varies, depending upon many factors such as the crop, the production season and the market demand. For example, in Brittany (France), commercial propagation of cauliflowers may involve trays containing approximately $700 \mathrm{seeds} / \mathrm{m}^{2}$ in winter seedling production and trays with over $1,300 \mathrm{seeds} / \mathrm{m}^{2}$ in summer seedling production (V. Faloya, personal communication). In the case of organic farming, diseases caused by soil- and seedborne fungal plant pathogens such as Rhizoctonia solani Kühn and Pythium spp. are a major problem for plant propagators, especially for high volume/high value plants such as vegetable Brassicas $(7,19)$. More generally, although it may play a very important role in regulating dynamics of disease in agricultural ecosystems, the link between host density and disease invasion remains poorly understood. Though a few notable studies in the past isolated and quantified host density as a factor in disease ecology (see the

Corresponding author: S. Poggi; E-mail address: sylvain.poggi@rennes.inra.fr

First and second authors contributed equally to this work.

* The $\boldsymbol{e}$-Xtra logo stands for "electronic extra" and indicates that Figures 1, 4, 5, 6, and 7 appear in color online.

http://dx.doi.org/10.1094/PHYTO-02-13-0033-R

(C) 2013 The American Phytopathological Society in replicated propagation systems with different planting densities, and fit a percolation-based epidemiological model to the data using Bayesian inference methods. The estimated probability of pathogen transmission between infected and susceptible plants is used to calculate the risk of invasion. By comparing the transmission probability and the risk values obtained for different planting densities, we are able to give evidence of a nonlinear relationship between disease invasion and the inter-plant spacing, hence to demonstrate the existence of a spatial threshold for epidemic invasion. The implications and potential use of our methods for the evaluation of disease control strategies are discussed.

Additional keywords: epidemiological modeling, field vegetables, invasion threshold.

review of Burdon and Chilvers [5] and references therein, and Madden and Boudreau [20]), with some focus upon soilborne diseases $(4,8,30)$, many fundamental questions remain open. In particular, while it is generally acknowledged that high seedling density may be at greater risk from soilborne disease spread, surprisingly little is known about the critical densities above which the risk increases substantially.

The risk of invasion by nearest-neighbor spread through a population of static hosts is recognized as a percolation problem $(9,14,22,32)$. Percolation theory has received increasing attention as a model for epidemic outbreak, notably for the identification of invasion thresholds $(2,9,13,25,29)$. Experimental validation for epidemics of plant disease conducted in artificial replicated microcosms confirmed the existence of a threshold for invasion $(2,13$, 25). The threshold is determined by the transmissibility of a pathogen (defined as the probability that an infectious host transmits infection to a neighbor). The threshold, in turn, depends upon the spacing between hosts on a lattice, and can be equated with the bond percolation threshold for the system by identifying lattice edges with pathogen transmission between neighbors (2,23-25). Yet, although statistical methods based on nearest-neighbor spread are already widely used in botanical epidemiology (28), the application of percolation theory has to date remained far from real agronomic systems.

Here, we apply a percolation-based method to the description and risk assessment of disease spread in a horticultural system. We focus on damping-off and wire-stem disease caused by the soilborne fungal plant pathogen, $R$. solani, on a population of cauliflower seedlings in commercial propagation trays. The principal questions we aim to address are as follows. Is there a critical host density above which the risk of pathogen invasion increases markedly? Can we assess and compare the risks of invasion for different host densities? 
We set up an experimental system comprising replicated host populations in propagation trays with different densities of cauliflower hosts. Each population was exposed to centrally placed inoculum of $R$. solani. The subsequent spread of disease was monitored and mapped. The effect of host density on the invasive behavior of the pathogen is analyzed using a percolation model in which transmissibility is an explicit parameter. Model parameters are estimated from successive snapshots of the spread of disease by means of Bayesian inference, and following Perez-Reche et al. (27) we derive a method to assess the risk of invasion for different host densities based on the posterior distribution of the transmissibility. We use the estimated risk of invasion to investigate the existence of a percolation threshold for pathogen invasion. The potential impact on disease management is discussed.

\section{MATERIALS AND METHODS}

Percolation and pathogen invasion. The percolation paradigm $(14,18,32)$ is a useful framework to describe pathogen invasion and associated thresholds for spatially explicit diseases. It is most efficient in the description of SI- and SIR-type diseases spreading among lattice-based populations (where S, I, and $\mathrm{R}$ refer to susceptible, infected, and removed hosts, respectively). The paradigm has been successfully validated in microcosm experiments $(2,23,25)$. In the bond percolation problem (32), a probability $p$ is given for any pair of neighboring sites on a regular lattice to be in contact (that is, for the bond joining the two sites to be "open"). As $p$ increases, clusters of communicating sites grow in number and size, and a percolating cluster, spanning the whole system (e.g., connecting all the edges of the lattice [32]), appears when $p$ is greater than a lattice-dependent "percolation threshold" $p_{c}\left(p_{c}=\right.$ 0.5 for a square lattice [16]). Although such threshold behavior is rigorously defined only for infinite systems, it can still be observed in relatively small systems as a sharp transition around $p_{c}$.

The problem of epidemic invasion in epidemiological models where a pathogen is stochastically transmitted from infected (I) hosts to susceptible (S) neighboring hosts on a lattice (with infected hosts possibly being removed, $\mathrm{R}$, after some time period) can be formulated as a bond-percolation problem $(14,18)$. The probability of pathogen transmission between neighboring hosts, the transmissibility $T$, is identified with the bond probability $p$, and disease patches at the end of the epidemic outbreak coincide with clusters of sites communicating via open bonds. The chance that the pathogen will finally invade the host population (i.e., that it reaches the edges of the system) is in turn related to the size of the spanning cluster, and depends only on the value of the transmissibility, regardless of the detailed temporal dynamics of pathogen transmission $(14,18)$. In infinite systems, the following threshold behavior is observed: when $T$ is smaller than the bond percolation threshold $p_{c}$ (henceforth, the invasion threshold $T_{c}$ ), the pathogen will fail to invade; when $T>T_{c}$, there is a non-zero probability that the pathogen will invade. As shown in Figure 1, a similar behavior emerges when the system size is finite.

In Figure 1A and B, we give two examples of epidemic outbreaks starting from the central site of a square lattice, and spreading by nearest-neighbor transmission with probability $T<T_{c}(T=$ 0.45 , Fig. 1A) and $T>T_{c}(T=0.55$, Fig. 1B). The final disease patch is shown in both cases, with black bonds marking the path of infection of the pathogen. The patch fails to invade the system for $T<T_{c}$ (Fig. 1A), while invasion can occur for $T>T_{c}$ (Fig. 1B shows a successful attempt). In Figure $1 C$, the probability of epidemic invasion, $P_{i n v}$, is plotted as a function of $T$ for two different system sizes, showing the threshold behavior of the system as a sharp increase of $P_{i n v}(T)$ around $T_{c}$. Such behavior is clear both for a very large system $(200 \times 200$ sites $)$ and for a smaller system $(20 \times 20$ sites, comparable with the experimental systems described below), although the transition is smoother (i.e., the increase is markedly less steep) in the latter case.

When dealing with soilborne diseases, environmental factors such as temperature and soil structure (26) can affect the value of the transmissibility. Our focus here is instead on the dependence of $T$ on the inter-plant distance (or equivalently, the planting density), a fundamental and controllable agricultural characteristic. Such dependence, also known as probability profile, has been characterized experimentally for several host-pathogen systems, by analyzing infection between pairs of donor-recipient hosts placed at different distances (e.g., by Bailey and Gilligan [1] or Kleczkowski et al. [17] for $R$. solani on radish seedlings). The transmissibility was usually found to decrease with distance $(1,17)$; as a consequence, $P_{i n v}(T)$ is also expected to decrease with distance. The signature of a percolation-type threshold effect is a strongly nonlinear change in $P_{i n v}$ around planting distances corresponding to values of $T$ close to $T_{c}$. Such nonlinear behavior has been found in model microcosm systems $(2,25)$.

Experimental design. The spread of disease was examined for the soilborne fungal plant pathogen $R$. solani spreading from a

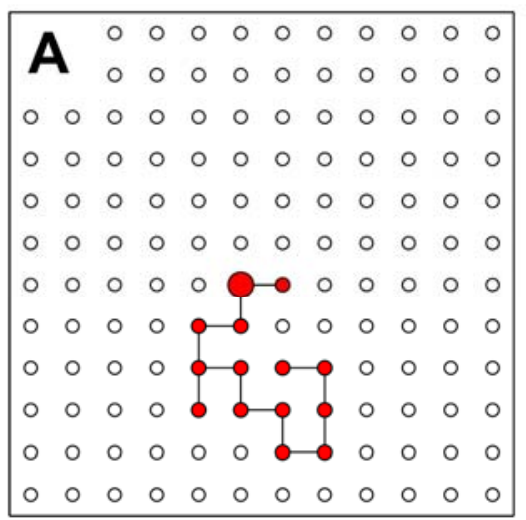

$\mathrm{T}=0.45$ (no invasion)

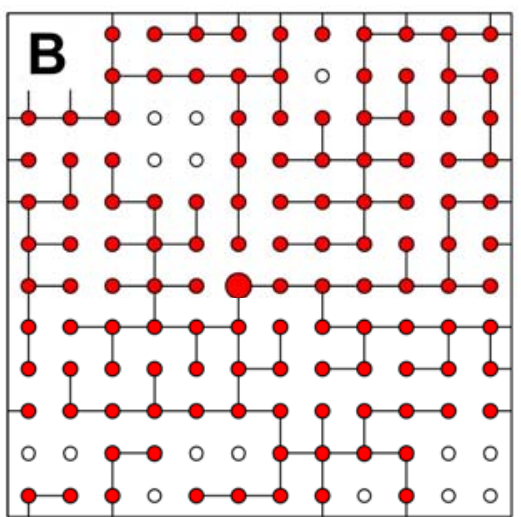

$\mathrm{T}=0.55$ (invasive spread)

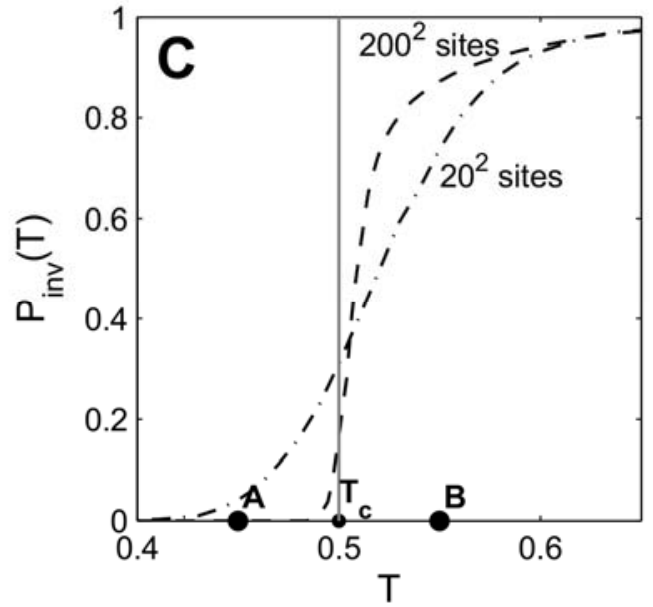

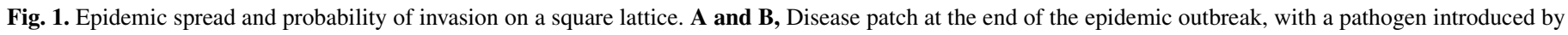

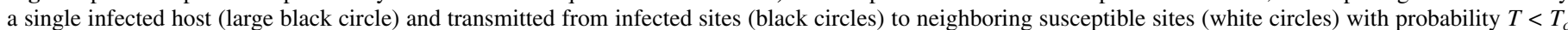

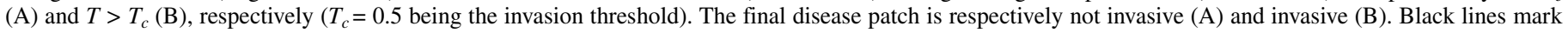

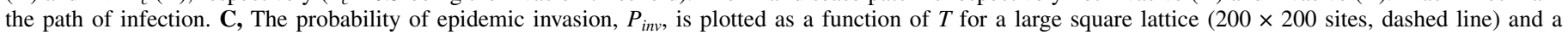

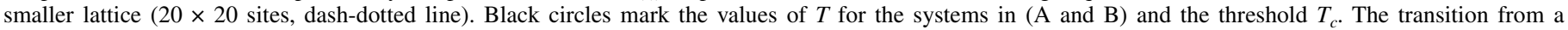

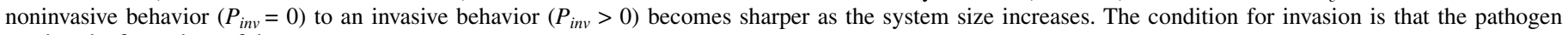
reaches the four edges of the system. 
single site in different densities of cauliflower (Brassica napus 'Skywalker') seedlings. Experiments were performed in a climatic chamber. Environmental conditions were set up for a $16 \mathrm{~h}$ photoperiod $/ 8 \mathrm{~h}$ dark period, at a temperature of $23^{\circ} \mathrm{C}($ day $) / 15^{\circ} \mathrm{C}$ (night), and hygrometry at $65 \%$. Such conditions are less variable, but are broadly similar, e.g., to those used by commercial growers in Brittany, France, in summer (V. Faloya, personal communication). Hosts were arranged on a square lattice in commercial propagation trays, measuring $60 \times 40 \mathrm{~cm}^{2}$, at five different densities (treatments), corresponding to inter-plant spacings of 22, 26, 34,44 , and $54 \mathrm{~mm}$ (Table 1). Each tray accommodated two square lattices, isolated by removing compost from a row of cells across the center of the tray. There were 10 replicate lattices per treatment. Inoculum ( $R$. solani Ag2-1, IMI 385769) consisted of discs of mycelium, $1.0 \mathrm{~mm}$ in diameter, which were removed from 5-day-old colonies grown in the dark at $23^{\circ} \mathrm{C}$ on Millipore filters and overlaying potato dextrose agar. Seedlings were sown to a depth of $10 \mathrm{~mm}$ in compost (Levingtons F1, $10 \%$ tap water by weight). Trays were fully randomized and disease (damping-off or wire-stem) in each square lattice was initiated by the infection of the central plant with four discs of mycelium; seedlings were mapped for disease symptoms on a daily basis for 27 days after inoculation.

Epidemiological model. Disease spread was described (Fig. 2) by a stochastic compartmental SI model $(3,13)$, whereby each host on the square lattice can be either susceptible (S) or infectious (I). We distinguish between primary infection that was restricted to infection of the central plant by introduced inoculum (X) and subsequent cycles of secondary infection (Fig. 2). One replicate for $54 \mathrm{~mm}$ spacing failed to establish any primary infection and is excluded from the analyses, which are predicated on the assumption that there is at least one infected seedling in the system.

Secondary infection occurs from an infected plant (donor) to one or more of its susceptible nearest neighbors (recipients). If the donor is infected at time $t_{d}$, pathogen transmission to a given recipient occurs at a time $t_{d}+t$, where the time $t$ is a random variable with a given distribution.

Transmissibility $(T)$ is defined as the probability of transmission ever occurring between pairs of neighboring hosts and is here assumed to be the same for all such pairs. Transmission from the central plant is described by a rescaled Gamma distribution:

$$
f_{1}(t)=T \frac{\tau^{-\chi}}{\Gamma(\chi)} t^{\chi-1} \exp (-t / \tau)
$$

where $T$ is the transmissibility, $\tau$ is a scale parameter (with units of time), and $\chi$ is a shape parameter.

Secondary transmission from any other infected plant occurs according to a rescaled Weibull distribution, as in Neri et al. (23), with the same value of $T$ as for primary infection:

$$
f_{2}(t)=T \frac{\kappa}{\lambda}(t / \lambda)^{\kappa-1} \exp \left(-(t / \lambda)^{\kappa}\right)
$$

where $\lambda$ is the time scale and $\kappa$ is a shape parameter.

TABLE 1. Key features of plant populations in propagation trays (two populations per tray): interplant spacing, the corresponding host population size (i.e., number of plants sown), and planting density ${ }^{a}$

\begin{tabular}{lcc}
\hline & \multicolumn{2}{c}{ Populations in propagation trays } \\
\cline { 2 - 3 } Interplant spacing & Number of plants & Planting density \\
\hline $22 \mathrm{~mm}$ & 169 & 2,066 plants $/ \mathrm{m}^{2}$ \\
$26 \mathrm{~mm}$ & 121 & 1,480 plants $/ \mathrm{m}^{2}$ \\
$33 \mathrm{~mm}$ & 49 & 918 plants $/ \mathrm{m}^{2}$ \\
$44 \mathrm{~mm}$ & 25 & 516 plants $/ \mathrm{m}^{2}$ \\
$54 \mathrm{~mm}$ & 25 & 343 plants $/ \mathrm{m}^{2}$ \\
\hline
\end{tabular}

a Densities typically used by commercial growers fall within the range considered here (e.g., 700 and 1,300 plants $/ \mathrm{m}^{2}$ for the French market).
Experimental data (Results) strongly suggested the choice of the two distinct dynamics in equations 1 and 2 . In principle, there is no biological argument favoring our choice of a model with a common value for $T$ in equations 1 and 2 against a model with two different values ( $T_{1}$ and $T_{2}$ in equation 1 and equation 2 , respectively). Indeed, we anticipate in the notation for equations 1 and 2 that the two models were tested against each other and found essentially equivalent (Results). Given such equivalence, the model with a single value for $T$ was preferred because it leads to a simple and parsimonious risk criterion (described below).

Parameter estimation. Model parameters $\left(\boldsymbol{\theta}=[T, \tau, \chi, \lambda, \kappa]^{t}\right)$ were estimated by Bayesian inference methods (11). Thus given prior probability distribution for the parameters, $\pi(\boldsymbol{\theta})$, the posterior distribution $\pi(\boldsymbol{\theta} \mid \boldsymbol{d})$, defined as the probability distribution for $\boldsymbol{\theta}$ given the observed data $\boldsymbol{d}$ is provided by Bayes' formula, $\pi(\boldsymbol{\theta} \mid \boldsymbol{d}) \propto \pi(\boldsymbol{\theta}) P(\boldsymbol{d} \mid \boldsymbol{\theta})$, where $P(\boldsymbol{d} \mid \boldsymbol{\theta})$ is the likelihood (the probability of the data given the parameters). For complex stochastic models, with many parameters, numerical methods are often needed to sample empirically from the posterior distribution. Here, we used Markov-chain Monte Carlo (MCMC) methods (11). Our algorithm and the related implementation follow closely those in Gibson et al. (12) and Neri et al. (23). Assume that the likelihood function for a given replicate $r, P\left(\boldsymbol{d}_{r} \mid \boldsymbol{\theta}\right)$, can be calculated analytically. A Markov chain is defined on the space spanned by the parameter vector $\boldsymbol{\theta}$ : each iteration of the chain involves proposing, then accepting or rejecting, a change (update) from the current parameter vector $\boldsymbol{\theta}^{c}$ (with prior $\pi\left(\boldsymbol{\theta}^{c}\right)$ and likelihood $P\left(\boldsymbol{d}_{\boldsymbol{r}} \mid \boldsymbol{\theta}^{c}\right)$ ) to a new vector $\boldsymbol{\theta}^{u}$ (with prior $\pi\left(\boldsymbol{\theta}^{u}\right)$ and likelihood $P\left(\boldsymbol{d}_{r} \mid \boldsymbol{\theta}^{u}\right)$ ). The update (Metropolis-Hastings algorithm) consists in changing the value of one of the parameters $T, \tau, \chi, \lambda$, and $\kappa$ in turn: the new parameter value is drawn from a normal distribution, centered on the current value, with suitably chosen width. The change is accepted with probability given by

$$
p_{a c c}=\min \left(1, \frac{\pi\left(\boldsymbol{\theta}^{c}\right) P\left(\boldsymbol{d}_{\mathbf{r}} \mid \boldsymbol{\theta}^{c}\right)}{\pi\left(\boldsymbol{\theta}^{u}\right) P\left(\boldsymbol{d}_{\mathbf{r}} \mid \boldsymbol{\theta}^{u}\right)}\right)
$$

Assuming that a few important conditions are satisfied (11), the Markov chain thus defined is guaranteed to converge to the posterior distribution $\pi\left(\boldsymbol{\theta} \mid \boldsymbol{d}_{\boldsymbol{r}}\right)$. These methods can be applied to analyze a set of replicates jointly simply by replacing the singlereplicate likelihood $P\left(\boldsymbol{d}_{r} \mid \boldsymbol{\theta}\right)$ by the joint likelihood $P_{\text {joint }}(\boldsymbol{d} \mid \boldsymbol{\theta})=$ $\Pi_{r} P\left(\boldsymbol{d}_{\boldsymbol{r}} \mid \boldsymbol{\theta}\right)$. In our case, an analytical expression for the likelihood $P\left(\boldsymbol{d}_{\boldsymbol{r}} \mid \boldsymbol{\theta}\right)$ was not available, due to data censoring, and model parameters were estimated by augmenting the parameter vector $\boldsymbol{\theta}$ to include the unknown infection times, prior to applying the algorithm described above: we refer the reader to Gibson et al. (12) for a detailed description of data augmentation methods in a similar framework.

Independent priors were used for all the parameters. A uniform prior in the interval $[0,1]$ was used for the transmissibility $T$, and exponential priors for the parameters $\lambda$ and $\kappa$ in equation 2 . The

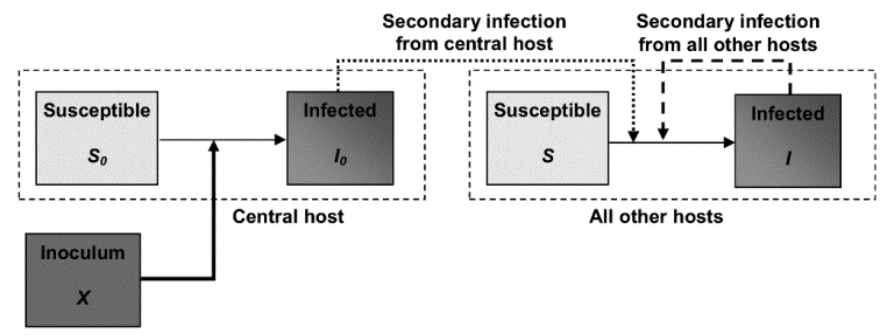

Fig. 2. Basic stochastic compartmental model describing our system. The central host (index 0 ) is infected by the inoculum $X$ (primary infection). The pathogen is then transmitted from an infected host (I) to a susceptible neighboring host (S) (secondary infection). We describe secondary infection from the central host (dotted arrow, equation 1) separately from secondary infection from all other hosts (dashed arrow, equation 2). 
time distribution for infection from the central plant (equation 1) was reparameterized in order to estimate the mean and standard deviation (given by $\chi \tau$ and $\chi^{1 / 2} \tau$, respectively) of the Gamma distribution in equation 1; exponential priors were used for both.

Model parameters were estimated for each individual replicate, and also jointly for all the replicates within the same treatment (i.e., planting density). Each chain was run for 200,000 Monte Carlo iterations, after a burn-in period of 20,000 iterations, in order to ensure that convergence had been reached. Goodness-offit was checked with two methods: by comparing predictive posterior distributions of disease progress with the empirical data; and by using the deviance information criterion (DIC [31]; the specific formulation used here corresponds to the $\mathrm{DIC}_{6}$ in Celeux et al. [6]).

Transmissibility and risk assessment. If the transmissibility of an experimental system can be estimated, the percolation-based description provides a powerful criterion to discriminate between systems where the pathogen (and hence disease) will potentially invade with high probability and systems that can be considered "safe" from invasion. Such a criterion is used here to compare the probability of invasion amongst different planting densities, using posterior distributions obtained by fitting jointly to all the replicates for a given treatment.

We also calculate a quantitative risk index for invasion for each individual replicate, following methods first proposed by PerezReche et al. (27). For a particular replicate, given the associated posterior distribution for the transmissibility, $\pi(T \mid \boldsymbol{d})$, the risk is defined as the integral over $T$ of the probability of invasion, $P_{i n v}(T)$, weighted by the posterior:

$$
\text { Risk }=\int_{0}^{1} \pi(T \mid \boldsymbol{d}) \mathrm{P}_{\mathrm{inv}}(T) d T
$$

The function $P_{i n v}(T)$ depends on the system size; however, in a large system, $P_{i n v}(T)=0$ for $T \leq T_{c}$, and $P_{i n v}(T)$ approaches 1 very quickly for $T>T_{c}$. Hence, we adopt the approximation $P_{i n v}(T)=1$ for $T>T_{c}$, so that the expression for the risk reduces to the area under the posterior curve for $T$ in the interval $\left[T_{c}, 1\right]$ :

$$
\text { Risk }=\int_{T_{c}}^{1} \boldsymbol{\pi}(T \mid \boldsymbol{d}) d T
$$

For very large systems, equation 3 overestimates invasion risk, at worst, by a small factor of the order of a few percent. The ap-

\begin{tabular}{|l|l|l|l|l|l|l|l|l|l|l|l|l|}
\hline & & & & & & & & & & & & \\
\hline & & & & & & & & & & & & \\
\hline & & & & & 27 & 27 & 27 & 27 & & & & \\
\hline & & & & & 25 & 25 & 25 & 27 & 27 & & & \\
\hline & & & & 25 & 24 & 24 & 24 & 25 & 25 & 27 & & \\
\hline & & & 26 & 25 & 23 & 21 & 23 & 23 & 24 & 27 & & \\
\hline & & & 27 & 21 & 21 & 6 & 24 & 24 & NA & 27 & & \\
\hline & & & & 25 & NA & 26 & 26 & 27 & 27 & & & \\
\hline NA & & & & 27 & 24 & 25 & 26 & & & & & \\
\hline & & & & 27 & 24 & 24 & 25 & 27 & & & & \\
\hline & & & & & 25 & 25 & 26 & & & & & \\
\hline & & & & & & & & & & & & \\
\hline & & & & & & & & & & & & \\
\hline
\end{tabular}

Fig. 3. A disease patch of damping-off of cauliflower seedlings grown within a commercial propagation tray. Inter-seedling distance: $22 \mathrm{~mm}$ (replicate 2). All cells are occupied by a host plant. Numbers refer to the date of appearance of disease symptoms (number of days after inoculation). NA stands for a nonemerged plant. The gray cell marks the central inoculated host. proximation was satisfactory also for small systems (numerical results not shown here), as overestimation for $T>T_{c}$ was roughly balanced by $P_{i n v}(T)$ being positive for $T \leq T_{c}$. A given treatment can be characterized by the distribution of individual risk index values across all the replicates. This method was also used in the present paper, together with the analysis of posterior distributions from pooled replicates.

\section{RESULTS}

Spread of damping-off disease. Damping-off disease began to appear after 5 days creating disease patches of variable size amongst the treatments (Fig. 3) until day 27 when observations stopped. Primary infection of the central seedling succeeded in 49 replicates out of 50, with damping-off symptoms occurring mostly between days 5 and 6 (Figs. 3 and 4). Symptoms associated to a first secondary infection were observed between days 14 and 27 (median close to 19 days, first and third quartiles close to 16 and 21 days, respectively), in 10 replicates out of 10 for the $22 \mathrm{~mm}$ inter-seedling spacing (resp. 9, 7, 3, and 1 replicates out of 10 for the $26,33,44$, and $54 \mathrm{~mm}$ spacings).

The temporal dynamics of the replicate epidemics are summarized for each host density in Figure 4. Disease spread rapidly in all trays at the highest planting density, i.e., with an interseedling distance of $22 \mathrm{~mm}$ (Fig. 4A), creating patches ranging from 8 to 48 plants (with a mean approximately equal to 20). Disease incidence dynamics was also investigated by fitting expo-
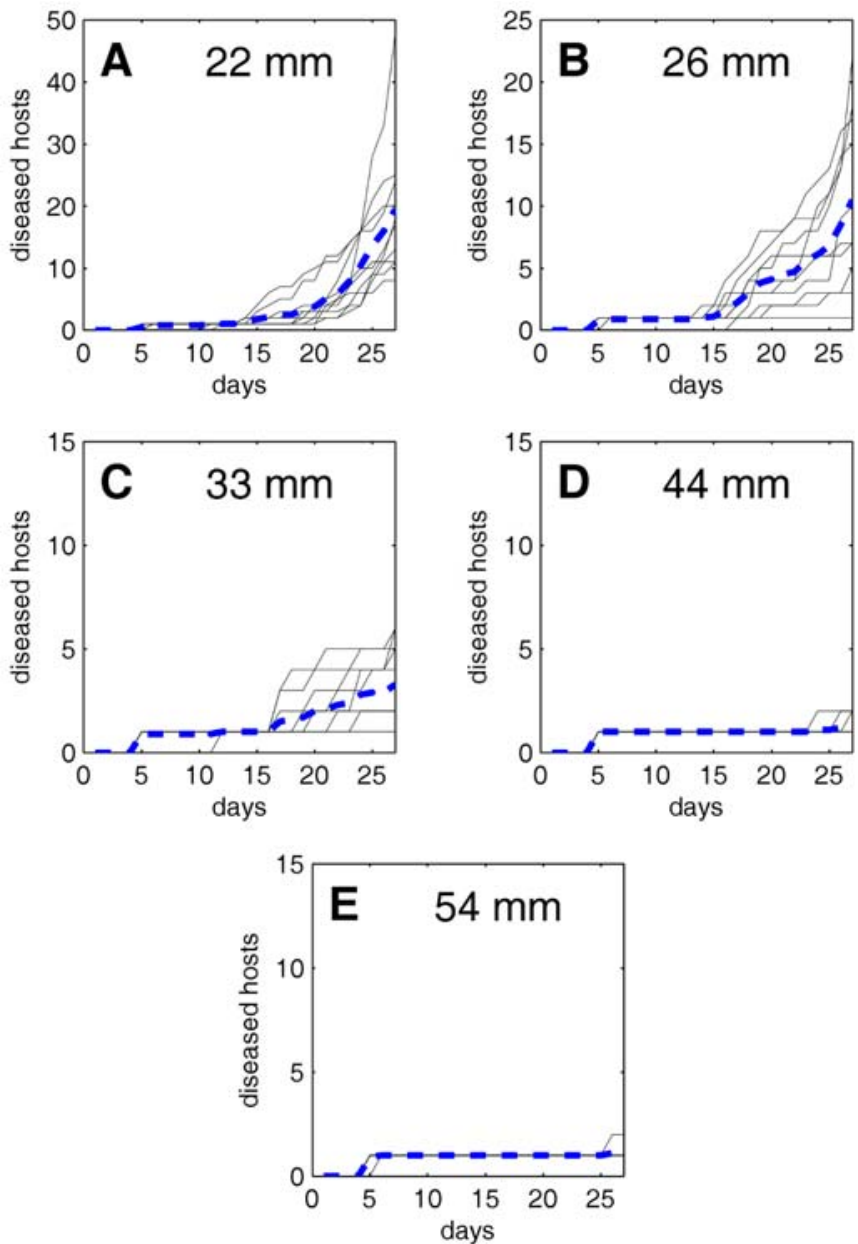

Fig. 4. Change in the number of diseased seedlings over time for replicate epidemics with inter-seedling spacing of 22, 26, 33, 44, and $54 \mathrm{~mm}$. For each treatment, the thin black disease progress curves are related to the 10 replicates, and the thick dashed line is the mean disease progress curve calculated over the 10 replicates. 
nential and quadratic functions to each replicate data in Figure 4 (fitting curves not shown), and analyzing first and second derivative of each function. In particular, for the treatment with $22 \mathrm{~mm}$ inter-seedling distance it was found that the rate of disease incidence was still accelerating (the second derivative of the fitting curve was positive) after 27 days (Fig. 4A).

For inter-seedling distances of 26 or $33 \mathrm{~mm}$ (Fig. 4B and C), the net rate of disease incidence continued to accelerate in some replicates but declined in others. Patch sizes at day 27 ranged from 1 to 22 with a mean of 10.5 diseased plants for the $26 \mathrm{~mm}$ treatment. The corresponding range for the $33 \mathrm{~mm}$ spaced plants was 1 to 6 plants with a mean of 3.5 diseased plants. There was negligible disease spread at $44 \mathrm{~mm}$ (Fig. 4D) and even less for $54 \mathrm{~mm}$ spacing (Fig. 4E), therefore this latter treatment is excluded from further analysis.

Parameter estimation and model performance. The fiveparameter model described by equations 1 and 2 was justified by the observation of two clearly distinct transmission dynamics. Transmission from the central cell occurred at longer, but tightly distributed, time scales (possibly due to slower build-up of inoculum on the seedling, initially very small), while further transmission events were characterized by shorter time scales. The five model parameters $(T, \tau, \chi, \lambda$, and $\kappa)$ were estimated from spatiotemporal disease observations for each replicate, and also jointly
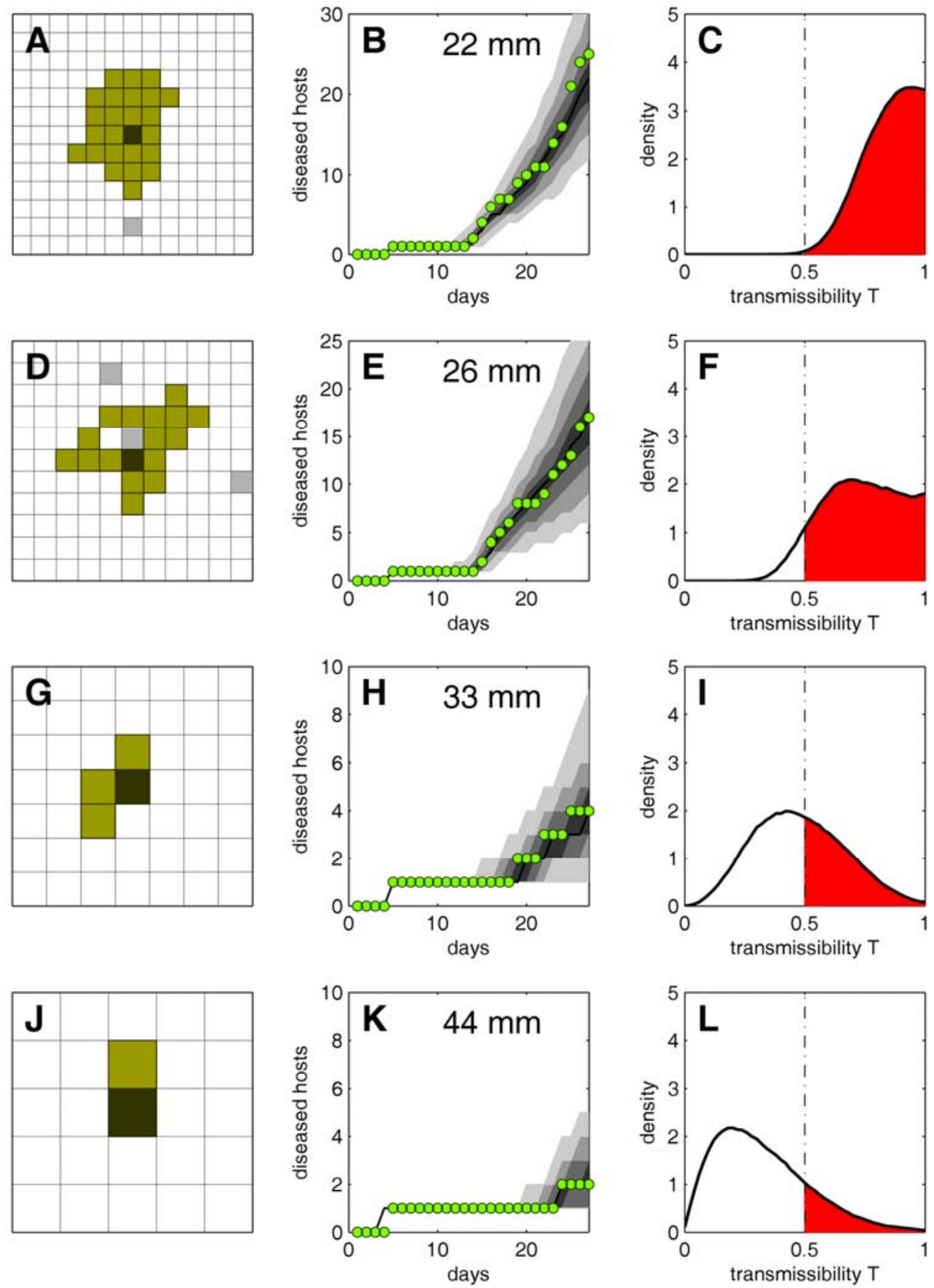

Fig. 5. Model parameters inference from spatiotemporal disease maps for four single replicates with inter-seedling spacing of A, B, and C, $22 \mathrm{~mm}, \mathbf{D}, \mathbf{E}$, and F, $26 \mathrm{~mm}, \mathbf{G}, \mathbf{H}$, and $\mathbf{I}, 33 \mathrm{~mm}$, and $\mathbf{J}, \mathbf{K}$, and $\mathbf{L}, 44 \mathrm{~mm}$. The first column represents the disease patches at the end of the experiment: each small square is a cell occupied by a seedling, and is black for the central (inoculated) host, medium gray for the other symptomatic hosts, and white for the asymptomatic hosts (light gray color indicates nonemerged plants). The second column compares the experimental disease progress curves (circles) with the distribution of simulated disease progress curves: the black line is the median of the distribution, while the grayscale shaded regions correspond to the 20\% (darker), $40 \%$, 60\%, and $80 \%$ (lighter) percentiles around the median. The third column gives the posterior distributions for the transmissibility: system invasiveness is characterized by the area under the distribution between $T=0.5$ and $T=1$ (equation 3), here highlighted in gray. 
for all the replicates of each treatment (i.e., associated with a particular planting density). For brevity, only estimates for the parameter $T$ are presented here, as they are essential in the identification of percolation-based thresholds (Materials and Methods and below). In Figure 5, we show results obtained for four single replicates, each associated to a different treatment, alongside the maps of disease patches at the end of the experiment (Fig. 5A, D, $\mathrm{G}$, and $\mathrm{J}$ ). Goodness-of-fit was assessed by comparing the observed data with simulated distributions for disease progress obtained using numerical simulations with parameters sampled from the posterior distributions. For all 40 replicates, simulations show good agreement with the observed disease progress curves: all the experimental curves lie consistently within the $95 \%$ credible interval of the predicted progress throughout the epidemic, and about half of the curves lie within the $50 \%$ credible interval (examples in Fig. 5B, E, H, and K). Posterior distributions for $T$ are shown in Figure 5C, F, I, and L, where the area contributing to the risk index (equation 3 ) is also highlighted.

Posterior predictive distributions for epidemics were also used to compare competing models, together with $\mathrm{DIC}_{6}(6,31)$. In
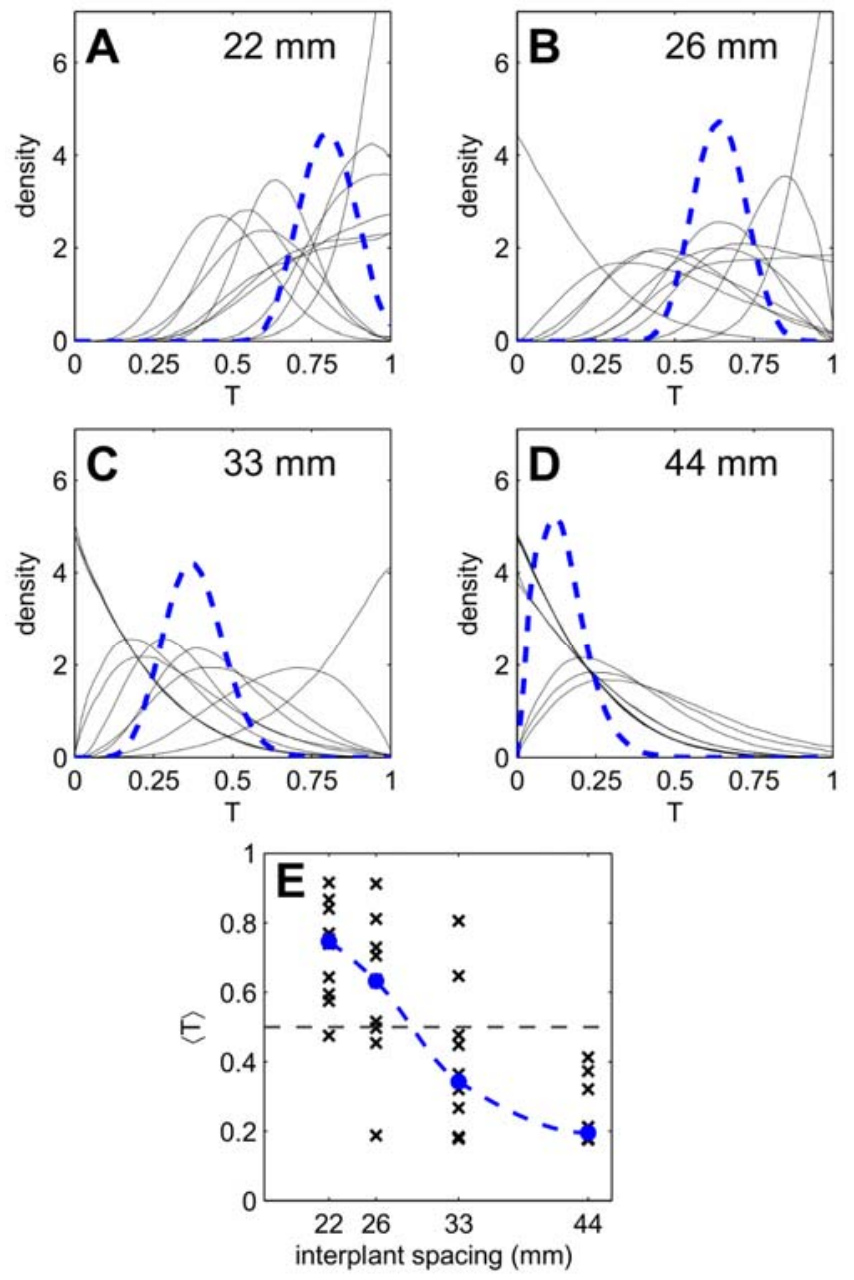

Fig. 6. Change in transmissibility with inter-seedling spacing. A to D, Posterior distributions for pathogen transmissibility, calculated for each replicate (thin black lines) and for all replicates pooled (thick dashed lines), for host densities corresponding to inter-seedling spacing of 22, 26, 33, and $44 \mathrm{~mm}$ (from A to D). E, summary statistics for estimated values of the transmissibility. The mean values of $T$ for individual replicates (black $\times$ ), calculated from the posteriors in (A to D), plotted as a function of the treatment interplant spacing. For each treatment, the median of the distribution of $\langle\mathrm{T}\rangle$ values is marked by a black circle (the dashed line is a guide for the eye). The invasion threshold, $T_{c}=0.5$, is marked by the horizontal line: using the dashed line as a guide, the corresponding value of the inter-plant spacing lies between 26 and $33 \mathrm{~mm}$. particular, we investigated the validity of our choice of a model with a single parameter for the transmissibility (equations 1 and $2)$, by comparison with a model with two distinct parameters $\left(T_{1}\right.$ and $T_{2}$ in equation 1 and equation 2, respectively). Both DIC scores and posterior predictive distributions (not shown here) show no significant advantage of the model with two parameters for $T$ with respect to the original model. Hence, the latter model can be considered a good choice, given that it allows a simple risk assessment criterion (equation 3).

Spatial invasion thresholds and risk assessment in propagation trays. The invasiveness associated with each planting density (corresponding to inter-plant spacing of 22, 26, 33, and 44 $\mathrm{mm}$ ) was investigated using posterior distributions for transmissibility for the individual replicates (thin black lines in Fig. 6A, B, $\mathrm{C}$, and D). Comparison of individual replicate distributions with posterior densities fitted jointly to the replicates (dashed curves in Fig. 6A, B, C, and D) confirms the high degree of heterogeneity in epidemic behavior amongst replicates within a given treatment. There is, however, a consistent trend in the reduction of transmissibility as the distance between plants on a lattice increases. The trend is summarized in Figure 6E, where the estimated mean values of $T$ for all individual replicates are plotted as a function of the treatment inter-plant spacing, and the median of the distribution of such values is used as the representative value of $T$ for each treatment. According to percolation theory, the transition between systems with high and low probability of invasion occurs when the transmissibility is decreased below the invasion threshold, $T_{c}=0.5$ (Materials and Methods). In our experimental system, such decrease occurs as the planting distance is increased from 26 to $33 \mathrm{~mm}$ (Fig. 6E; the two distributions, black crosses, were significantly different according to Wilcoxon rank-sum test, with a $P$ value of 0.026 ). Thus, the spatial invasion threshold can be located between 26 and $33 \mathrm{~mm}$.

The switch between high-risk and low-risk regimes can be investigated using the risk index (equation 3) calculated from the posterior distribution for $T$ for each individual replicate. In Figure 7 , the distribution of risk values for each treatment is plotted (black crosses) as a function of the inter-plant spacing. The risk of invasion is very high at the highest planting density $(22 \mathrm{~mm}$ spacing), being between 0.75 and 1 in $70 \%$ of the replicates. Although still relatively high, the risk decreases significantly for propagation systems with $26 \mathrm{~mm}$ spacing: only $50 \%$ of replicates are subject to a risk of invasion $>0.75$, but the risk is still greater than 0.5 in $60 \%$ of the replicates. The switch occurs for lower

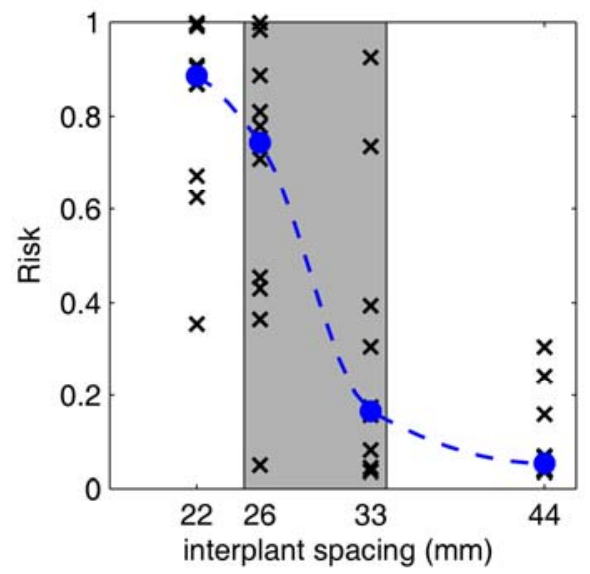

Fig. 7. Change in risk of invasion with inter-seedling spacing: summary statistics for the risk index. Values of the risk index for individual replicates (black $x$ ) are plotted against the inter-plant spacing. For each treatment, the median of the distribution of risk values is marked by black circles (the dashed line is a guide for the eye). The sharp drop of the risk median between 26 and $33 \mathrm{~mm}$ (gray area) occurs when the median value of $\langle\mathrm{T}\rangle$ in Figure $6 \mathrm{E}$ crosses the invasion threshold. 
planting densities, with $20 \%$ of replicates showing a risk greater than 0.5 and $60 \%$ below 0.25 for propagation systems characterized by a $33 \mathrm{~mm}$ inter-seedling distance. This shift towards low risk of invasion is even stronger for $44 \mathrm{~mm}$ spacing. The median of the within-treatment distribution (filled circles in Figure 7) displays a sharp drop between the 26 and $33 \mathrm{~mm}$ spacings, with a decrease of $\approx 0.6$ in value (cf. the smoother behavior of $T$ in the same region in Figure $6 \mathrm{E}$, where the decrease in $T$ is $\approx 0.3$ ). As discussed in the Materials and Methods (Fig. 1C), such a nonlinear response of the probability of invasion around $T_{c}=0.5$ is consistent with the existence of a spatial invasion threshold as predicted from percolation theory.

\section{DISCUSSION}

Spatial invasion threshold. In this paper, a percolation framework is used to describe the threshold behavior for invasion of damping-off epidemics of $R$. solani in cauliflower populations in commercial seedling propagators. The potential for pathogen invasion in those systems was investigated by combining epidemiological modeling, Bayesian inference for parameter estimation, and a percolation-based definition of the invasion threshold. We particularly focus on transmissibility, defined as the probability that an infectious host transmits infection to a susceptible neighbor, and from which we derive a risk index for invasion. All the methods introduced here can be directly applied (9) to the more general case in which an infected host can be removed (become immune or die) with a given probability or rate (SIR models $[3,13])$.

We estimated pathogen transmissibility between pairs of infected and susceptible hosts for a range of host densities in propagation systems corresponding to inter-seedling spacing of 22, 26, 33 , and $44 \mathrm{~mm}$. We show that there is a decreasing trend in transmissibility as inter-plant spacing increases (summarized in Figure $6 \mathrm{E}$ by the decrease of median values of $T$ within each treatment). An invasion threshold for the pathogen was identified between 26 and $33 \mathrm{~mm}$ inter-plant distance, where the estimated transmissibility was close to the critical value $T_{c}=0.5$ for percolation on a square lattice (Fig. 6E).

The relative frequencies of invasion risk were derived using estimates of transmissibility for individual replicates (Fig. 7), based on a method for risk assessment introduced by Perez-Reche et al. (27), and using a convenient, readily calculable approximating formula (equation 3). A plot of the risk index as a function of the treatment inter-plant spacing (Fig. 7) shows a sharp switch from high (22 and $26 \mathrm{~mm}$ inter-seedling spacing) to low risk (33 and $44 \mathrm{~mm}$ spacing), consistent with the existence of a spatial invasion threshold. While this behavior has been shown before for experimental model systems $(2,23,25)$, to our knowledge, this is the first time that the existence of a percolation threshold has been demonstrated for pathogens in a real agronomic setting.

Implications for disease management. Although there is evidence for variability in transmissibility amongst replicates within host densities (Fig. 6A, B, C, and D, thin lines), we observe a significant shift from the high-risk regime (high values for transmissibility) to the low-risk regime (low values for transmissibility) as planting density decreases. A natural question follows: which systems should we protect preferentially? If we assume that the application of a control reduces the probability of pathogen transmission (i.e., the associated distribution for $T$ is shifted to the left), disease control is then most effective for systems close to the invasion threshold, where even a small shift in transmissibility (i.e., a low effective treatment) is able to switch epidemic behavior from invasive to noninvasive. Within the present propagation systems, we would thus favor prophylaxis on manufactured trays with 26 and $33 \mathrm{~mm}$ inter-seedling spacing. In cases where transmissibility is low (cf. $44 \mathrm{~mm}$ spacing, Fig. 6D, thick line) and the system is essentially noninvasive, investing on prevention would be much less cost-effective. Conversely, when transmissibility is high (22 mm spacing, Fig. 6A, thick line), even a strong preventive effort would prove only partially effective. As risk measure plays a key role in the evaluation and adoption of control strategies (21), we posit that our risk assessment method could be useful in the decision process for disease management, combined with other economically relevant factors (e.g., lower densities imply a reduction in output, but can also improve vigor and quality of healthy seedlings).

Opportunities for control also exist by manipulating the spatial distribution of the seedling population. In the current work, seedlings were organized on a square lattice with a corresponding invasion threshold probability of $T_{c}=0.5$. Thresholds for invasion are known for a range of spatial architectures (32). For example, the threshold for a honeycomb lattice is 0.65 , which would correspond in our experimental system to an inter-seedling distance of approximately $26 \mathrm{~mm}$ (Fig. 6B). Hence, for a honeycomb lattice system with $33 \mathrm{~mm}$ inter-plant spacing there would be a negligible risk of pathogen invasion. This suggests that the selection of the lattice alone can provide protection against the likelihood of epidemic spread. Similarly, removal, isolation, or protection of a critical number of seedlings would provide control from the invasive spread of the pathogen (25).

We point out that the specific values of the transmissibility discussed here, hence the position of the threshold, could be influenced by the growing conditions used in our experiment (depending, e.g., on temperature, soil type, and light; see Materials and Methods; see Otten et al. (26) for the effect of soil structure on $T$ ). Given a system with different growing conditions, the methods proposed here could then be used to determine the threshold and the risk of invasion in situ from a few replicated trays, and in turn to minimize the risk for those specific conditions.

\section{ACKNOWLEDGMENTS}

This paper was submitted after the premature and tragic death from cancer of our colleague Doug Bailey. We are very grateful to Doug for his deep insight and long-term guidance that were central to the development of this project. We thank J. Filipe for useful discussions. This research was supported by the INRA "Plant Health \& Environment" Division, and the French Research Agency (ANR) through funding of the SYSBIOTEL project referenced ANR-08_STRA-14. C. A. Gilligan also gratefully acknowledges the support of a BBSRC Professorial Fellowship.

\section{LITERATURE CITED}

1. Bailey, D. J., and Gilligan, C. A. 1997. Biological control of pathozone behavior and disease dynamics of Rhizoctonia solani by Trichoderma viride. New Phytol. 136:359-367.

2. Bailey, D. J., Otten, W., and Gilligan, C. A. 2000. Saprotrophic invasion by the soil-borne fungal plant pathogen Rhizoctonia solani and percolation thresholds. New Phytol. 146:535-544.

3. Bailey, N. T. J. 1975. The Mathematical Theory of Infectious Diseases. 2nd ed. Griffin, London.

4. Burdon, J. J., and Chilvers, G. A. 1975. Epidemiology of damping-off disease (Pythium irregulare) in relation to density of Lepidum sativum seedlings. Ann. Appl. Biol. 81:135-143. doi:10.1111/j.1744-7348. 1975.tb00530.x

5. Burdon, J. J., and Chilvers, G. A. 1982. Host density as a factor in plant disease ecology. Annu. Rev. Phytopathol. 20:143-166.

6. Celeux, G., Forbes, F., Robert, C. P., and Titterington, D. M. 2006. deviance information criteria for missing data models. Bayesian Analysis 1:651-673.

7. Clarkson, J., and Roberts, S. 2011. Disease Management in Organic Brassica Seed and Transplants. Warwick HRI, Coventry.

8. Converse, R. H., and Stace-Smith, R. 1971. Rate of spread and effect of Tomato ringspot virus on red raspberry in the field. Phytopathology 9:1104-1106.

9. Davis, S., Trapman, P., Leirs, H., Begon, M., and Heesterbeek, J. A. P. 2008. The abundance threshold for plague as a critical percolation phenomenon. Nature 454(7204):634-637.

10. DEFRA. 2011. Basic Horticultural Statistics 2011. Department for Environment, Food and Rural Affairs, UK. 
11. Gelman, A., Carlin, J. B., Stern, H. S., and Rubin, D. B. 2003. Bayesian Data Analysis. 2nd ed. Chapman \& Hall/CRC Press, Boca Raton, FL.

12. Gibson, G. J., Otten, W., Filipe, J. A. N., Cook, A., Marion, G., and Gilligan, C. A. 2006. Bayesian estimation for percolation models of disease spread in plant populations. Stat. Comp. 16:391-402. doi:10.1007/s11222-0060019-z

13. Gilligan, C. A. 2002. An epidemiological framework for disease management. Pages 1-64 in: Advances in Botanical Research, Vol 38. Academic Press Ltd., London.

14. Grassberger, P. 1983. On the critical behavior of the general epidemic process and dynamical percolation. Math. Biosci. 63:157-172.

15. Hutin, C. 2009. Le diagramme de la distribution 2008: Le tableau de bord de la filière. Infos-Ctifl 255:16-21.

16. Kesten, H. 1980. The critical probability of bond percolation on the square lattice equals 1/2. Commun. Math. Phys. 74:41-59.

17. Kleczkowski, A., Gilligan, C. A., and Bailey, D. J. 1997. Scaling and spatial dynamics in plant-pathogen systems: From individuals to populations. Proc. Roy. Soc. London Series B-Biol. Sci. 264(1384):979-984.

18. Kuulasmaa, K. 1982. The spatial general epidemic and locally dependent random graphs. J. Appl. Prob. 19(4):745-758. doi:10.2307/3213827

19. Lammerts van Bueren, E. T., Struik, P. C., and Jacobsen, E. 2003. Organic propagation of seed and planting material: An overview of problems and challenges for research. NJAS Wageningen J. Life Sci. 51:263-277.

20. Madden, L. V., and Boudreau, M. A. 1997. Effect of strawberry density on the spread of anthracnose caused by Colletotrichum acutatum. Phytopathology 87:828-838. doi:10.1094/phyto.1997.87.8.828

21. McRoberts, N., Hall, C., Madden, L. V., and Hughes, G. 2011. Perceptions of disease risk: From social construction of subjective judgments to rational decision making. Phytopathology 101:654-665. doi:10.1094/ phyto-04-10-0126

22. Mollison, D. 1977. Spatial contact models for ecological and epidemic spread. J. R. Stat. Soc. B 39:283-326.
23. Neri, F. M., Bates, A., Fuchtbauer, W. S., Perez-Reche, F. J., Taraskin, S. N., Otten, W., Bailey, D. J., and Gilligan, C. A. 2011. The effect of heterogeneity on invasion in spatial epidemics: From theory to experimental evidence in a model system. PLoS Comp. Biol. 7(9). doi:e100217410.1371/journal.pcbi.1002174

24. Neri, F. M., Perez-Reche, F. J., Taraskin, S. N., and Gilligan, C. A. 2011. Heterogeneity in susceptible-infected-removed (SIR) epidemics on lattices. J. Roy. Soc. Interface 8(55):201-209. doi:10.1098/rsif.2010.0325

25. Otten, W., Bailey, D. J., and Gilligan, C. A. 2004. Empirical evidence of spatial thresholds to control invasion of fungal parasites and saprotrophs. New Phytol. 163(1):125-132. doi:10.1111/j.1469-8137.2004.01086.x

26. Otten, W., Hall, D., Harris, K., Ritz, K., Young, I. M., and Gilligan, C. A. 2001. Soil physics, fungal epidemiology and the spread of Rhizoctonia solani. New Phytol. 151:459-468.

27. Perez-Reche, F., Neri, F., Taraskin, S. N., and Gilligan, C. 2012. Prediction of invasion from the early stage of an epidemic. J. Roy. Soc. Interface 9(74):2085-2096.

28. Real, L. A., and McElhany, P. 1996. Spatial pattern and process in plant-pathogen interactions. Ecology 77(4):1011-1025. doi:10.2307/ 2265572

29. Salkeld, D. J., Salathe, M., Stapp, P., and Jones, J. H. 2010. Plague outbreaks in prairie dog populations explained by percolation thresholds of alternate host abundance. Proc. Natl. Acad. Sci. USA 107(32):1424714250. doi:10.1073/pnas.1002826107

30. Scott, M. R. 1956. Studies of the biology of Sclerotinium cepivorum Berk. II. The spread of white rot from plant to plant. Ann. Appl. Biol. 44(4):584-589. doi:10.1111/j.1744-7348.1956.tb02156.x

31. Spiegelhalter, D. J., Best, N. G., Carlin, B. R., and van der Linde, A. 2002. Bayesian measures of model complexity and fit. J. Roy. Stat. Soc. Ser. B-Stat. Method. 64:583-616. doi:10.1111/1467-9868.00353

32. Stauffer, D., and Aharony, A. 1994. Introduction to Percolation Theory. 2nd Revised ed. Taylor \& Francis, London. 